\title{
LAS ESPECIALIDADES EN ENFERMERÍA EN CHILE: SITUACIÓN ACTUAL Y SU REGULACIÓN
}

\author{
Paulina Milos Hurtado \\ Mg. Derecho Público \\ Enfermera, Abogado \\ E-mail: paulina.milos@gmail.com
}

http://dx.doi.org/10.7764/Horiz_Enferm.29.3.173-179

\section{INTRODUCCIÓN}

El desarrollo de la Enfermería en Chile, desde sus inicios, se vincula a las necesidades más sentidas de la sociedad, en que la profesión ha contribuido a dar respuesta a las demandas en salud de la población; con carencia de recursos y muchas veces de apoyo institucional ha posibilitado una prestación de cuidados coherente con las necesidades sociales en evolución.

Es así como, en la década de 1920, las enfermeras hospitalarias centraban su atención en las personas con enfermedades infecto-contagiosas; y las enfermeras comunitarias, en la mujer embarazada, el recién nacido y las personas con tuberculosis. Y, actualmente se presentan como un recurso profesional idóneo para combatir el cáncer desde su prevención ${ }^{1}$.

En el ámbito de la formación, en el año 1906, la Enfermería inicia su etapa profesional con la creación de la primera escuela universitaria, anexa a la Universidad de Chile. La que se consolida el 30 de junio del año 1921, fecha en que el presidente Arturo Alessandri Palma decreta que las escuelas de enfermería establecidas en los hospitales que cumplieran disposiciones, tales como: condiciones de admisión, presentación de planes y programas oficiales, e infraestructura adecuada para la formación profesional, fueran instituciones que otorgaran el título de enfermera universitaria. Esta acción revela que es el Estado chileno el que sitúa a la profesión Enfermera en un estatus universitario, el más alto nivel educacional de la época².

De este modo, las enfermeras (os) han ido conformando su rol social centrado en las necesidades de cuidado, biomédicas y de protección del entorno de las personas; en los diferentes niveles de atención, en personas sanas y enfermas, a lo largo del ciclo de vital.

\footnotetext{
${ }^{1}$ Ministerio de salud Subsecretaría de Salud Pública, Plan Nacional del Cáncer 2018 - 2028. Disponible en: https://cdn.digital.gob.cl/filer_public/d3/0a/d30a1f5e-53d9-4a31-a4fee90d8d9a2348/documento_plan_nacional_de_cancer.pdf

${ }^{2}$ Consultar: Flores, R. (1965). Historia de la Enfermería en Chile (Vol. 1). Santiago, Chile: Universidad de Chile; Godoy, M., Ortiz, Y., Fardella, N. (1951). Desarrollo y perspectiva de la Enfermería Sanitaria en Chile. Boletín de la Oficina Sanitaria Panamericana, XXXI (2), 158-164; Pincheira, S: Educación de Enfermería. Enfermería. 1965; (5):21-23, 1980; Núñez, E. (2012). Disertación Tesis Doctoral "Enfermeras chilenas 1970-1980: dos décadas de transformación de la identidad, un legado para la memoria”. Universidad Andrés Bello. Santiago Chile.
} 


\section{DE LA LEGITIMACIÓN A LA LEGISLACIÓN}

El Código Sanitario, en su Libro V "De la Medicina y las Profesiones Afines", hace reserva legal de las funciones y ámbito del ejercicio de las profesiones de la salud; bajo esta fórmula abierta, subyace la idea de equipo de salud, con la que se pretende promover la corresponsabilidad en el logro de los fines comunes y en la mejora de la calidad de la atención.

En este contexto, en el año 1997, el legislador reconoce que las (os) enfermeras(os) cumplen una función social, esto es que resuelven un problema social, aportando un bien esencial, específico y valioso a la atención en salud, que se constituye en un bien digno de protección jurídica.

Al efecto, el ejercicio profesional de las(os) enfermeras(os), bajo la denominación "servicios profesionales de la enfermera", es recogido en el artículo 113, inciso cuarto del Libro V, prescribiendo que "Los servicios profesionales de la enfermera comprenden la gestión del cuidado en lo relativo a la promoción, mantención y restauración de la salud, la prevención de enfermedades o lesiones, y la ejecución de acciones derivadas del diagnóstico y tratamiento médico y el deber de velar por la mejor administración de los recursos de asistencia para el paciente".

De tal modo, el rol social de la enfermera (o) comprende tres grandes funciones vinculadas entre sí: i) la gestión del cuidado en lo relativo a promoción, mantención y restauración de la salud, la prevención de enfermedades o lesiones; ii) la ejecución de acciones derivadas del diagnóstico y tratamiento médico; y iii) el deber de velar por la mejor administración de recursos de asistencia para el paciente ${ }^{4}$.

\section{EL DESARROLLO DISCIPLINAR}

El cuidado integral de la persona y su entorno, en su estado de salud y enfermedad, ha sido el motor del desarrollo disciplinar en Enfermería. Por una parte, los problemas que deben enfrentar en su quehacer diario demandan una formación general basada en el conocimiento amplio que atraviesa varias disciplinas. Y, por otra, cada vez es más imperioso profundizar en la disciplina propia, dado el cambio del perfil epidemiológico de la sociedad chilena que se observa con un aumento de necesidades de cuidados de enfermería en los extremos de la vida, el avance tecnológico y la evidencia científica en que se sustenta su actuar.

La Enfermería constituye un amplio campo de investigación. En Chile, la capacidad de las enfermeras y los enfermeros se puede inferir a partir del Informe "Sobre indicadores

\footnotetext{
${ }^{3}$ El término "afines", en igual sentido, es utilizado bajo el vocablo "análogas" en el Código penal chileno ver: MILOS HURTADO, Paulina. Las profesiones "análogas" en el delito de ejercicio ilegal de la medicina del artículo 313 a) del código penal chileno: propuesta para su determinación. RDUCN [online]. 2012, vol.19, n.1, pp. 197-239

${ }^{4}$ Sobre la función social de la (o) Enfermera (o): MILOS H, Paulina; BORQUEZ P, Blanca y LARRAIN S, Ana Isabel. LA "GESTIÓN DEL CUIDADO" EN LA LEGISLACIÓN CHILENA: INTERPRETACIÓN Y ALCANCE. Cienc. enferm. [online]. 2010, vol. 16, n.1, pp. 17-29. ISSN 0717-9553. Disponible en: http://dx.doi.org/10.4067/S071795532010000100003.
} 
cienciométricos de la actividad científica chilena" (Conicyt, 2013) ${ }^{5}$, que da cuenta de la producción científica del país. De acuerdo con los datos entregados por Conicyt, la disciplina de Enfermería (Nursing) cuenta con una categoría propia que aporta al esfuerzo investigador del país un número significativamente mayor de publicaciones que las que hace el conjunto de las denominadas "HealthProfessions" o Profesiones de la Salud.

La presencia de la Enfermería como una disciplina de carácter autónomo y diferenciado en los indicadores cienciométricos presentados se relaciona con la "Propuesta de Norma Práctica para encuestas de Investigación y Desarrollo Experimental” o "Esquema de Disciplinas Frascati” (OCDE, 2006) ${ }^{6}$, que asigna a la Enfermería un código propio en el área de Ciencias de la Salud. Este manual contiene las definiciones básicas y categorías de las actividades de Investigación y Desarrollo, y ha sido aceptada por científicos de todo el mundo.

La modalidad de formación reflexiva y crítica en Enfermería se reafirma con el grado de Licenciado iniciado en 1981 en la Universidad de Talca, y alcanza su máxima expresión con el primer Doctorado en Enfermería en la Universidad de Concepción, creado en 2004. En la actualidad, el desarrollo disciplinar de la Enfermería en Chile se traduce en dos Programas de Doctorado en Enfermería y más de 34 programas de Magíster y Especializaciones ${ }^{7}$.

En concordancia existen órganos de difusión científica tales como las revistas Ciencia y Enfermería (SciELO), Horizonte de Enfermería (Index, Cuiden) y Benessere (Latindex y Red Iberoamericana de Edición Científica en Enfermería) editadas bajo el alero de la Universidad de Concepción, de la Pontificia Universidad Católica de Chile y de la Universidad de Valparaíso, respectivamente.

En suma, la Enfermería se encuentra inserta en las ciencias de la salud y desde hace muchas décadas constituye una compleja disciplina científico-humanista con un objeto, conocimientos, lenguaje y actos propios. Su desarrollo configura áreas de estudio y conocimiento específico, en constante profundización y progreso.

\section{LAS ESPECIALIZACIONES/ESPECIALIDADES EN ENFERMERÍA}

La evolución de la Enfermería ha estado íntimamente unida al concepto saludenfermedad de cada época histórica. Las “especializaciones” en Enfermería se han ido dando a la par de los cambios epidemiológicos, tecnológicos y sociales, constituyéndose en un compromiso y un desafío profesional. ${ }^{5}$ CONICYT, SCIMAGO LAB: Principales indicadores cienciométricos de la actividad científica chilena 2011, Informe
2013. Madrid-Valparaíso, 2013, p.135,140. Disponible en: http://www.conicyt.cl/wp-
content/uploads/2013/08/Informe_2013_baja_resolucion.pdf
${ }^{6}$ Disponible en: http://www.conicyt.cl/fondecyt/files/2013/03/Disciplinas-OCDE.pdf
${ }^{7}$ No obstante que el grado de Licenciado en Enfermería se otorgue en la totalidad de las universidades, en forma previa a
la obtención del título profesional, este requerimiento no se encuentra establecido por ley. Actualmente se encuentra en
trámite Proyecto de Ley (Boletín $N^{\circ} 10.678-04$ ) sobre "Licenciatura en Enfermería" y "Exclusiva formación universitaria".

2018, Horiz Enferm,29,3,173-179 
A partir de los años 60 del siglo pasado, surgen las primeras comunidades científicas en Enfermería abocadas a un área específica que demanda de cuidados especializados; los que requieren de estudios más allá de la formación básica general propio de la profesión.

Es en estas circunstancias, en que motivadas por las propias enfermeras (os) surgen las Sociedades Científicas, en Enfermería ${ }^{8}$.

Y tal como ha sido el devenir histórico aquellas han ido recogiendo las necesidades sociales y avances tecnológicos de cada época. Solo a modo de ejemplo, se pueden mencionar la Sociedad de Enfermería en Salud Pública, que, al tiempo de su constitución, concentra sus esfuerzos en reducir las tasas morbimortalilidad, verdadero flagelo nacional; la Sociedad Chilena de Enfermería en Cardiología y Cirugía Cardiovascular coetánea al estudio de Framinghan (FraminghamHeartStudy), la cirugía cardíaca y avance tecnológico; la Sociedad Chilena de Enfermería en Diálisis y Trasplante Renal reconocida por su aporte en el alto grado de seguridad que han alcanzado estas modalidades de tratamiento, en nuestro país, o la recientemente constituida "Sociedad Chilena de Enfermería en Oncología"9.

En el momento epidemiológico actual, es un imperativo, que el desarrollo observado en las especializaciones de enfermería en el ámbito biomédico se expanda a otras de sus funciones como lo es la "Gestión del Cuidado" y la "Administración de los recursos de asistencia”. En este último ámbito, las enfermeras (os) cuentan con la formación básica para responder a las nuevas tendencias que se perfilan en el ámbito de las políticas públicas en salud, tales como la "atención centrada en el paciente"; "acceso universal (y oportuno) a la atención en salud"; "calidad y seguridad en la prestación de servicios de salud", "sostenibilidad de la atención en salud" y otras de similar naturaleza.

El desarrollo del país y su compromiso con la protección de la salud de las personas demanda avanzar en Enfermería, de un estado de "especialización" de crecimiento inorgánico, al reconocimiento de las "especialidades" y la formación de "especialistas”, que formados bajo programas fundados, sistemáticos y evaluables; hagan énfasis en el aporte enfermera (o) a la humanidad, eficiencia y eficacia, seguridad y calidad, y sostenibilidad de la atención en salud.

\section{LA REGULACIÓN DE LAS ESPECIALIDADES EJERCIDAS POR LOS PRESTADORES INDIVIDUALES DE SALUD}

La Ley $19.937^{10}$, sobre Autoridad Sanitaria, asignó al Ministerio de Salud el deber de "establecer un sistema de certificación de especialidades y subespecialidades de los prestadores individuales de salud legalmente habilitados para ejercer sus respectivas profesiones $^{11}$, y a la Superintendencia de Salud (SIS), la función de mantener registros

\footnotetext{
${ }^{8}$ Las Sociedades Científicas de Enfermería, son asociaciones de derecho privado, sin fines de lucro, que se rigen por las normas del Título XXXIII del Libro Primero del Código Civil, por las disposiciones contenidas en la Ley N. ${ }^{\circ} 20.500$ sobre Asociaciones y Participación Ciudadana en la Gestión Pública, y por sus respectivos Estatutos, debidamente aprobados. ${ }^{9} \mathrm{El}$ Colegio de Enfermeras de Chile en su página web, registra nueve sociedades científicas.

${ }^{10}$ Actualmente contenida en el Título I del DFL No 1 , Ministerio de Salud, 2005.

${ }^{11} \mathrm{La}$ disposición se refiere a prestadores individuales de salud legalmente habilitados para ejercer sus respectivas profesiones, entre los que, conforme al Código Sanitario y al Registro de Prestadores Individuales de la SIS, queda comprendido el profesional enfermera(o).
}

2018, Horiz Enferm,29,3,173-179 
públicos de los prestadores individuales de salud, de sus especialidades y subespecialidades y de las entidades certificadoras; unido a los procesos de acreditación de los prestadores institucionales.

En la mencionada ley, se define la certificación como "el proceso por el que se reconoce que un prestador individual domina un cuerpo de conocimientos y experiencias relevantes en un determinado ámbito del trabajo asistencial, otorgando el correspondiente certificado".

El propósito del sistema de certificación, es garantizar la calidad de las acciones de salud, entregando un sello distintivo a los profesionales que posean las competencias necesarias para otorgar ciertas y determinadas prestaciones. Esto mediante: i) las Entidades Certificadoras, las que aplican mecanismos y procedimientos homogéneos de evaluación de conocimientos teórico y prácticos y, ii) la SIS manteniendo un registro público que contenga información fidedigna, acerca de la condición profesional (título profesional, título de especialista y subespecialista) de los prestadores individuales de salud ${ }^{12}$.

Bajo el marco regulatorio actual, las especialidades y subespecialidades de Enfermería, en tanto ejercidas por enfermeras (os) prestadores individuales de salud habilitados por un título profesional para ejercer sus respectivas profesiones, deben ser reguladas por la Autoridad Sanitaria, quedando adscritas al "Sistema de certificación y recertificación de especialidades y subespecialidades"; teniendo como mandato constitucional el Artículo 19 N99, sobre el derecho a la protección de la salud de las personas.

Los programas de formación de especialistas responden a una Norma Técnica Operativa, que se acuerda entre el Ministerio de Salud, y un grupo de expertos de la especialidad a regular, con requisitos de contenidos y destrezas, que las Entidades Certificadoras, deberán exigir se les demuestre mediante evaluaciones teóricas y prácticas.

La Certificación es requisito para la inscripción en el Registro de Prestadores Individuales de Salud, transparentado con ello el sistema de especialidades en salud; a la vez que garantiza la idoneidad en la formación de los especialistas registrados.

Las entidades docente-asistenciales, que impartan los programas de especialidades en Enfermería, deberán estar acreditados, de modo de asegurar la uniformidad y calidad del proceso enseñanza-aprendizaje; con el fin, entre otros, de favorecer la formulación de políticas públicas e investigación de problemas nacionales de salud.

La actualización de la especialidad o subespecialidad de Enfermería deberá ser evaluada, en el tiempo, conforme a los cambios sociales, epidemiológicos y tecnológicos. A su vez, con el fin de asegurar la calidad y seguridad de la atención en Enfermería, se deberá aplicar el sistema de recertificación previsto para los prestadores individuales de salud, a

\footnotetext{
${ }^{12} \mathrm{El}$ sistema de certificación de especialidades que actualmente regula las especialidades médicas, odontológicas, farmacéuticas y bioquímicas se constituye básicamente por: i) El Reglamento de certificación de las especialidades de los prestadores individuales de salud y de las entidades que las otorgan, D.S No 8, Ministerio de Salud, 2013; ii) Norma Técnica General No 145, Ministerio de Salud, relativa a las Normas Técnicas Operativas Generales de la Certificación de Especialidades y Subespecialidades. Esta norma, en el año 2013, fue modificada con la finalidad de incorporar la descripción de "aspectos técnicos y conocimientos mínimos" para la certificación de las especialidades y subespecialidades médicas y odontológicas, reconocidas a la fecha ${ }^{12}$.y iii) El Registro Nacional de Prestadores Individuales de Salud, con base legal en la Ley $\mathrm{N}^{\circ} 19.937$.
} 
través de mecanismos y modalidades de validación periódica de las especialidades reglamentadas por la Autoridad Sanitaria; entregando al Estado la responsabilidad de dar fe pública sobre la condición profesional de los prestadores individuales (especialistas).

La obtención del título de especialista o subespecialista en el área de Enfermería, en tanto aquellas son ejercidas por enfermeras (os) prestadores individuales habilitados para ejercer sus respectivas profesiones, supone un nivel superior de cualificación profesional al acreditar una formación específica en algún ámbito concreto del ejercicio profesional.

De tal modo, el título de especialista, es un título oficial que autoriza a ejercer la especialidad en cualquier lugar del país que, además, es necesario para utilizar públicamente la denominación de especialista, sea en un servicio público o privado de salud. Si bien, legalmente, el título de especialista no es obligatorio es requisito exigido por los establecimientos de salud estar inscrito en el Registro Nacional de Especialidades de la Superintendencia de Salud, para ejercer la profesión como especialista, que si lo requiere. El título es garantía de idoneidad.

\section{CONSIDERACIONES FINALES}

La profesión enfermera se encuentra normada en el Código Sanitario Libro V, "De la Medicina y Profesiones Afines". De este modo, como profesión afín a la medicina, comparte la exigencia de un título profesional que emane de la Universidad de Chile u otra reconocida por el Estado y, consecuentemente le es aplicable la regulación que en materia de especialidades profesionales, la Autoridad Sanitaria ha aplicado a aquellas profesiones que el artículo 112 señala en forma expresa.

La complejidad del conocimiento que sustenta la intervención de las enfermeras (os) en la atención en salud ha exigido a las universidades, con carácter progresivo, una formación general sistemática, rigurosa en los ámbitos del ser, saber y hacer, que asegure a la población un servicio integral de la más alta calidad ${ }^{13}$, que comprenda los aspectos esenciales de la disciplina de enfermería y disciplinas afines; a la vez de desarrollar en sus egresados un pensamiento crítico que permita al profesional profundizar en áreas específicas.

Ante las demandas sociales las universidades, en virtud de la facultad de otorgar títulos propios, asesoradas por la $\mathrm{ACHIEEN}^{14}$ o las Sociedades Científicas de Enfermería o Médicas han asumido la formación de enfermeras especializadas en áreas críticas; sin una programación ni reconocimiento oficial. No obstante ello, esta formación ha contribuido, a que las enfermeras (os) especializados hagan un aporte significativo en mejorar la humanización, calidad científico-técnica y la seguridad en la atención de salud de las personas, contribución específica que ha permitido disminuir las tasas de morbimortalidad

\footnotetext{
${ }^{13}$ Cecilia Campos: Apuntes de clase "Enfermería Profesión y disciplina del cuidado" (Agosto 2008).

${ }^{14}$ Asociación de de Derecho Privado, sin fines de lucro, creada en el año 1963 cuya misión es velar por la calidad de la formación de las enfermeras y enfermeros de Chile, conformada por veintidós Unidades Académicas de Enfermería acreditadas y sus académicos. Entre sus objetivos destaca contribuir con los órganos del Estado, en materia de Salud y Educación, aportando resultados de estudios que justifiquen cambios curriculares en la formulación de programas de estudios.
}

2018, Horiz Enferm,29,3,173-179 
en áreas específicas y mejorar el bienestar integral del recién nacido, niños, adolescentes, adultos, personas mayores, dolientes y moribundos.

En este proceso hay que reconocer el trabajo conjunto entre médicos y enfermeras/os; con espíritu de equipo. Es frecuente observar que las actividades de difusión de los avances científicos, en cada una de las especialidades, se realicen en forma paralela. En el entendido que ambos estamentos, cada uno en su disciplina, se debe formar, actualizar e innovar con una visión y objetivos comunes. Las especialidades médicas requieren de su correlato en enfermería.

Las especialidades y subespecialidades son hoy una necesidad social. Esta modalidad de trabajo responde al avance tecnológico que permite cuidar, con base en la evidencia científica, calidad y seguridad teniendo como centro a la persona o grupo de personas portadores de determinadas necesidades de cuidados, cuadros o patologías que exigen de la enfermera (o), con posterioridad a la obtención del título profesional, adquirir nuevas competencias, bajo un programa de formación sistemática, teórico-práctico, que otorgue un título, el que, en Chile, deberá ser certificado por una universidad o una agencia certificadora, ambas entidades acreditadas y reconocidas por el Estado.

El desarrollo de las "especialidades en enfermería" constituye un aporte valioso, esencial y único, mediante el cual se resuelve un problema en un campo específico con conocimiento, competencias y habilidades que permiten dar una respuesta homogénea a las necesidades y derechos de las personas, tanto en el área de cuidados de enfermería, asistencial biomédica y administrativa.

Las características de la disciplina de Enfermería y de la profesión enfermera(o), componen una estructura clave del sistema de salud, al incidir en las variables críticas en salud: acceso, oportunidad, humanidad, calidad/seguridad, y sostenibilidad de la atención, la que se verá potenciada mediante la formación de especialistas y subespecialistas.

En atención a lo expuesto, la formación de enfermeras(os) especialistas, dada su relevancia para la sociedad es una cuestión de Estado que debe preocupar a la Autoridad Sanitaria. 